\title{
Effects of flattening the stockpile crest and of the presence of buildings on dust emissions from industrial open storage systems
}

\author{
C. Turpin \& J. L. Harion \\ Department of Industrial Energetics, Ecole des Mines de Douai, France
}

\begin{abstract}
On industrial sites, fugitive dust emissions from open storage systems for bulk materials, such as coal or iron, represent a significant part of overall estimated particle emissions and can lead to environmental and health risks. The aeolian erosion process depends strongly on the turbulent flow structure over the stockpiles and is significantly conditioned by the topography of the site, the wind direction and intensity and also the shape of the stockpiles. This paper presents wind flow structures obtained by three-dimensional numerical simulations for various stockpile configurations and for a global industrial site simulation with different wind directions. The first aspect investigated by these simulations is the effect caused by the flattening of the stockpile crest on dust emissions. This study provides information to industrials on the best geometrical pile characteristics of an oblong shape pile configuration in order to limit particles emissions. The second aspect investigated in this study is the wind exposure over stockpiles while considering its environment on an industrial site. This study highlights the influence of the presence of surrounding buildings or stockpiles on the real exposure of granular materials, and will allow a more accurate evaluation of fugitive dust emissions on industrial sites. Keywords: computational fluid dynamics (CFD), fugitive dust emissions, emission factors, wind erosion, flat-topped stockpiles.
\end{abstract}

\section{Introduction}

Investigations [1-3] about aeolian erosion concurred to state that the particles emissions from a pile of granular material strongly depend on the wind flow structure over the pile which depends itself on the topography of the studied site, the 
wind direction and the pile shape. The understanding of the flow interactions with the pile led to the estimation of fugitive dust emissions. In fact, the knowledge of the near surface velocity allows to estimate the particles quantity likely to take off.

To quantify fugitive dust emissions from stockpiles, most of manufacturers use a methodology based on the determination of an emission factor for each given source. The most common emission factor formulations for wind erosion is proposed by the US EPA (United States Environmental Protection Agency). The EPA emission factor EF for wind-generated particulate emissions is expressed in units of grams per year as follows [4]:

$$
E F=k \sum_{i=1}^{N} P_{i} S_{i}
$$

where $k$ is a particle size multiplier, $N$ the number of disturbances per year, $P_{i}$ an erosion potential corresponding to the observed (or probable) fastest mile of wind for the $i$ th period between disturbances in $\mathrm{gm}^{-2}$ and $S_{i}$ the pile surface area in $m^{2}$.

The erosion potential function for a dry exposed surface is :

$$
\begin{gathered}
P=58\left(u^{*}-u_{t}^{*}\right)^{2}+25\left(u^{*}-u_{t}^{*}\right) \quad \text { for } u^{*}>u_{t}^{*} \\
P=0 \quad \text { for } u^{*} \leq u_{t}^{*}
\end{gathered}
$$

where $u^{*}$, the wind friction velocity, is given by $u^{*}=0.1 u_{10}^{+}\left(u_{s} / u_{r}\right) . u_{t}^{*}$ is the threshold friction velocity $\left(m s^{-1}\right), u_{10}^{+}$the fastest mile value collected on a anemometer reference height of $10 \mathrm{~m}, u_{s}$ the wind speed measured at $25 \mathrm{~cm}$ from the piles surface and $u_{r}$ the wind speed reference measured at a height of $10 \mathrm{~m}$.

For large disturbances of wind, such as example on large storage piles, the emission factor is estimated by the sum of local erosion potentials corresponding to a same value of $u_{s}$. The emission factor in then given by:

$$
E F=k \sum_{i=1}^{N} \sum_{j=1}^{M}\left(P_{j} S_{j}\right)_{i}
$$

where $M$ is the number of area parts, $S_{j}$ is the corresponding surface area. This formulation allows the consideration of the influence of the pile geometry and the wind direction on the velocity distribution over the stockpiles by dividing the pile area into sub-areas of constant degree of wind exposure. The EPA's report provides cartographies, derived from wind tunnel studies, representing the wind exposure of two representative pile shapes [4].

Further studies [5-7], focusing on fugitive dust emissions on industrial sites, introduce a new approach to quantify the wind exposure over a stockpile by using data coming from Computational Fluid Dynamics simulations. Three-dimensional numerical simulations, previously validated against wind tunnel measurements, have been employed to simulate the wind flow structure over a wide variety of pile forms and dimensions that can be found on industrial sites. These simulations 
are used to extend the number of available wind exposure cartographies of stockpiles. The analysis of the results obtained for a wide variety of stockpile geometries allowed to suggest solutions to limit aeolian erosion. For example, an optimal stockpile aspect ratio [6] was proposed to reduce dust emissions.

This paper presents the results of two different studies carried out using the three-dimensional numerical simulations previously validated by [5]. In the first study, the flow structure over various flat-topped stockpiles was simulated in order to find the best clipping height on an oblong shape stockpile which could lead to a decrease of dust emission rate. This study was initiated from the assessment that stockpile crest is the area most subject to erosion, so it becomes relevant to simulate wind flow over different stockpiles having undergone a crest clipping. In the second part of this paper, the first results of the simulated wind flow structure over a real configuration of industrial site are presented. This study aims to highlight the necessity to take into account the presence of buildings surrounding the storage areas in the estimation of the fugitive dust emissions by wind erosion. Very few studies are dedicated to this type of complex configuration compared to isolated stockpile configurations.

\section{Numerical simulations description}

\subsection{Geometry, mesh and turbulence model definitions}

The commercial Computational Fluid Dynamics (CFD) software FLUENT was employed to simulate the wind flow over various pile configurations. Full verification and validation of numerical simulations to experimental results [8] was demonstrated and discussed by [5]. The numerical model was also validated for a full scale height. The dimensions of the calculation domain were defined according to the geometric characteristics of the stockpiles on the site and the incidence angle of the flow on the pile. Various tests were performed to ensure aerodynamical independence of the flow patterns over the tested piles as to the effect of the domain boundaries. Characteristic elements of the mesh are shown on Figure 1. An irregular mesh was applied to follow the shape of the geometry.

A grid sensitive test was realized to ensure that numerical results are independent of the grid. Profiles of velocity, turbulent kinetic energy and specific dissipation rate, which are specifics of an atmospheric boundary layer, were used to define the entry of calculation domain. Symmetry boundary conditions were used for the lateral sides and the upper limit of the domain. The lower boundary was considered as a wall. Turbulence closure model was achieved through application of the two-equation $k-\omega$ SST model [9]. This choice is based on a comparative study between experimental results and numerical results for different closure models and a single pile configuration. These validated numerical simulations can be used for various piles configurations, and their results associated to the formulation 1, 2 and 3 allow the estimation of the erosion potential for the configuration tested. 


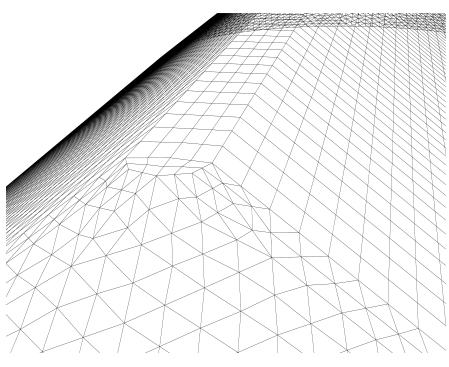

(a)

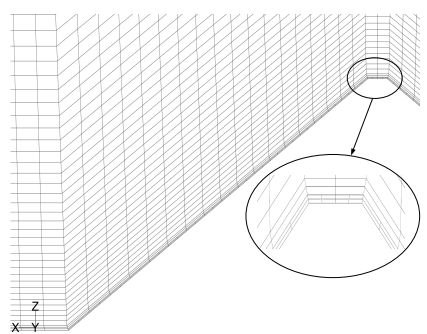

(b)

Figure 1: (a) Top view of the grid over the test 2, (b) transversal cut of the domain representing the grid around the test 2 .

\subsection{Tested configurations}

The numerical model, previously explained, was applied to simulate flow over various flat topped stockpiles heights and over a complete industrial site. The whole simulations were carried out for only one wind speed, $u=5 \mathrm{~ms}^{-1}$. Indeed it was shown that the normalized wind speed $u_{s} / u_{r}$ is independent of the wind speed flow [6]. For each configuration, data of normalized velocity values $u_{s} / u_{r}$, grouped in steps of 0.1 and their corresponding areas were computed at $25 \mathrm{~cm}$ from the piles surface. Results were then integrated in the EPA emission factor formulations to estimate the rates of dust emissions. A coal material was taken as a reference for the calculations, its threshold friction velocity was determined from a wind tunnel experiment and averaged about $u_{t}^{*}=0.35 \mathrm{~ms}^{-1}$.

\subsubsection{Flat topped piles}

Different configurations with various pile heights and wind conditions were simulated. The modeled piles had a constant volume, shape (oblong), and a constant side slope angle $\left(37^{\circ}\right)$, but different clipping heights. The characteristics of the pile without clipping correspond to the pile configuration determined by [6] as the one having the weakest erosion potential for various wind directions. Geometric properties of the tested configurations are reported in Table 1. The clipping heights applied to the stockpile ranges from 0 to $3 \mathrm{~m}$ and varies by step of $0.5 \mathrm{~m}$. The wind flow structure over two stockpile configurations was studied for four wind incidence angles : $\theta=0^{\circ}, 30^{\circ}, 60^{\circ}$ and $90^{\circ}$. Only one configuration of flat topped pile (test 4) was compared to the sharp crested pile configuration in order to limit the number of calculations.

\subsubsection{Complete industrial site}

The previously validated three-dimensional numerical simulations method was also used to simulate the wind flow over coal stockpiles on a power plant in 
Table 1: Geometric characteristics of the tested piles.

\begin{tabular}{|c|c|c|c|c|c|c|}
\hline Test & $\theta\left({ }^{\circ}\right)$ & Volume $\left(m^{3}\right)$ & Pile angle $\left({ }^{\circ}\right)$ & Height $(m)$ & Length $(m)$ & Width $(m)$ \\
\hline $1,8,10,12$ & $90,60,30,0$ & 31477 & 38 & 16 & 74,5 & 41.000 \\
\hline 2 & 90 & 31477 & 38 & 15,5 & 74,5 & 41.016 \\
\hline 3 & 90 & 31477 & 38 & 15 & 74,5 & 41.060 \\
\hline $4,9,11,13$ & $90,60,30,0$ & 31477 & 38 & 14,5 & 74,5 & 41.140 \\
\hline 5 & 90 & 31477 & 38 & 14 & 74,5 & 41.256 \\
\hline 6 & 90 & 31477 & 38 & 13,5 & 74,5 & 41.414 \\
\hline 7 & 90 & 31477 & 38 & 13 & 74,5 & 41.616 \\
\hline
\end{tabular}

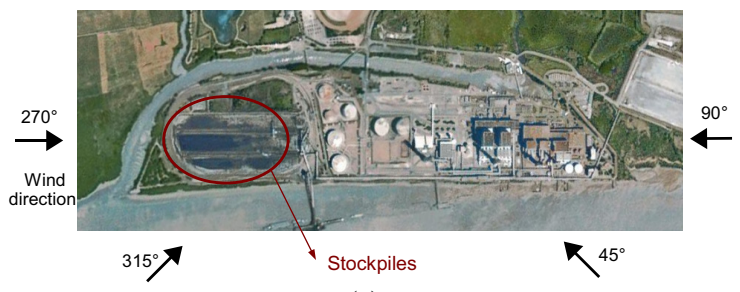

(a)

\begin{tabular}{ccc}
$\theta$ & $\begin{array}{c}\text { Complete } \\
\text { site }\end{array}$ & $\begin{array}{c}\text { Site with only } \\
\text { the stockpiles }\end{array}$ \\
\hline 270 & test 1A & test 1B \\
\hline 315 & test $2 \mathrm{~A}$ & test $2 \mathrm{~B}$ \\
\hline 45 & test $3 \mathrm{~A}$ & test 3B \\
\hline 90 & test $4 \mathrm{~A}$ & test 4B \\
\hline
\end{tabular}

(b)

Figure 2: (a) Top view of the industrial site, (b) Test names for the different configurations.

real conditions. Two configurations were tested for various wind flow directions: $\theta=270^{\circ}, 315^{\circ}, 45^{\circ}$ and $90^{\circ}$. The first configuration represents the site with only the stockpiles. The second one represents completely the site with the surrounding buildings. The various configurations tested are presented on Figure 2 and in Table 1.

\section{Results and discussion}

\subsection{Flat topped piles}

Qualitatively, the overall results are consistent with literature and experimental data $[1,10-12]$ : flow deceleration at the base of the pile, flow acceleration up the 


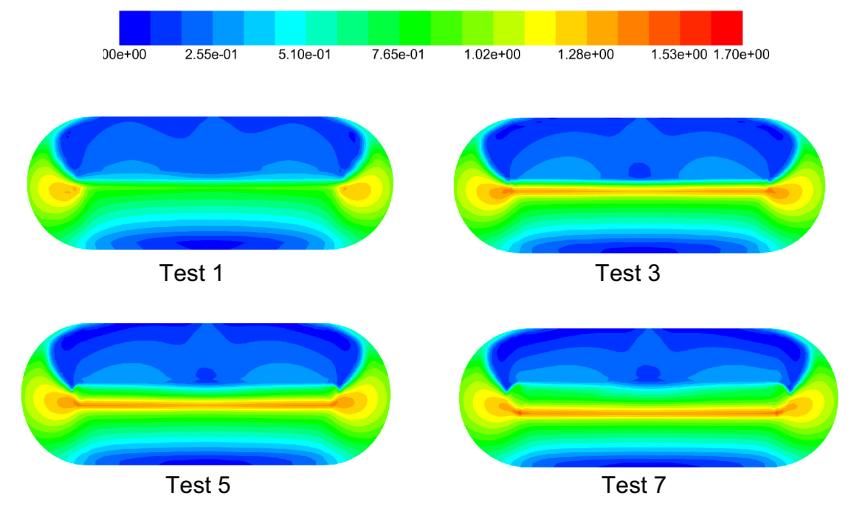

Figure 3: Top view of the normalized velocities contours $u_{s} / u_{r}$ at $25 \mathrm{~cm}$ from the pile surface for the tests $1,3,5$ and 7 .

windward slope and toward the sides, followed by a region of flow separation on the crest, and finally a low velocity region of reversed flow in the lee side.

The pile geometry effect on the wind speed near the pile surface is shown in Figure 3 through the contours of the normalized velocity $u_{s} / u_{r}$. On the windward slope of the stockpiles, where the flow is accelerated, the normalized velocity $u_{s} / u_{r}$ increases progressively from the toe to the top of the stockpile. Maximum $u_{s} / u_{r}$ value are then recorded at the top, and on the lateral sides of the stockpiles, what implies a high erosion potential in these zones. When the clipping height increases, the ratio $u_{s} / u_{r}$ is increasing, this happens mostly on the top of the flattopped stockpiles (Figure 3). Figures 4(a) and (b) show the velocity contours for the tests 1 and 4 . They show that the pile geometry near the crest has a very strong influence on the flow detachment. Figures 4(b) and (d) show, for flat topped pile configuration, a strong low pressure field, and much larger velocity values near the crest. Simulations predict a strongly negative pressure gradient on the first ridge of the flat topped pile in comparison with the sharp crested configuration (Figure $4(d))$. This negative pressure gradient justifies the fact that the flow acceleration up the stoss slope is higher on the flat topped pile configuration than in the sharp crested configuration.

After the flat top, the flow separates from the surface and creates a large recirculation. Figure 3 shows that the flow recirculation in the lee side induces a low velocity region which implies that the erosion process stops.

Figure 5 presents the emission factors calculated by the EPA method for the tests 1 to 7 for different wind speeds. It shows that the evolution of the emission factors for each configuration tested not depend on the wind velocity. For a clipping height between 0 and $1.5 \mathrm{~m}$, the calculated emission factors increase significantly. For a clipping height larger than $1.5 \mathrm{~m}$, the emission factors remain approximately constant in comparison with the test 4 . This is due to the fact that the pile's heights 


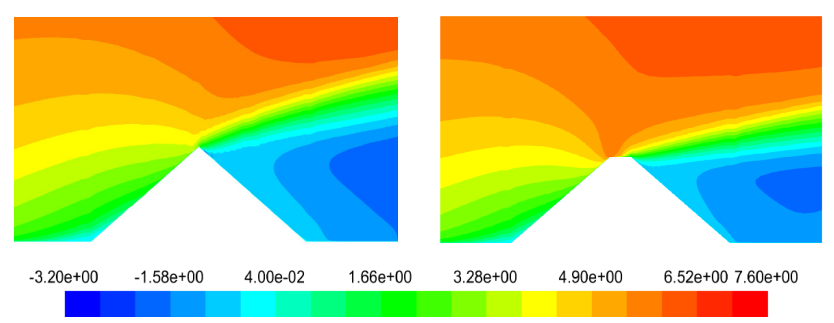

(a)

(b)

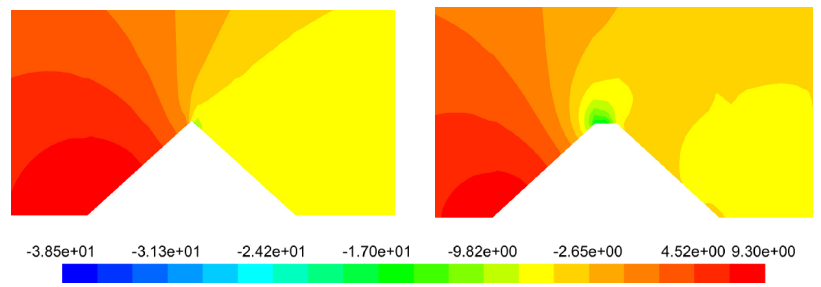

(c)

(d)

Figure 4: Transversal cut following the wind flow direction of the velocity contours (test 1 (a) and test 4 (b)) and the pressure contours (test 1 (c) and test $4(\mathrm{~d}))$.

are smaller, and therefore, the stoss slope areas subject to the aeolian erosion are reduced. Nevertheless, for these configurations, the maximum value of normalized velocity increases as the clipping height grows up, in particular at the approach of the stockpile flat top.

The contours of normalized velocity above the pile surfaces for the tests 1,4 and 8 to 13 for different velocity directions are shown on Figure 6. They reveal that when the wind incidence angle on the stockpiles $\theta$ varies from $90^{\circ}$ to $0^{\circ}$, the impact zone of the flow on the pile upwind and the recirculation zone on the leeward slope downstream are considerably modified and reduced. As a result, the areas of low normalized velocity become smaller to the advantage of regions subject to the aeolian erosion. These results provide important information on the influence of the wind direction on dust emissions.

The clipping of the stockpiles does not allow to reduce dust emission but, on contrary, increases the dust emission rates. Areas corresponding to erosion are systematically higher for the flat topped configuration. The configuration having the weakest potential erosion, among the tested configurations, is the sharped crest stockpile put perpendicularly to the wind flow.

\subsection{Complete industrial site}

In order to extend the number of cartographies representing the wind exposure of a stockpile available in the EPA report, three-dimensional numerical simulations 


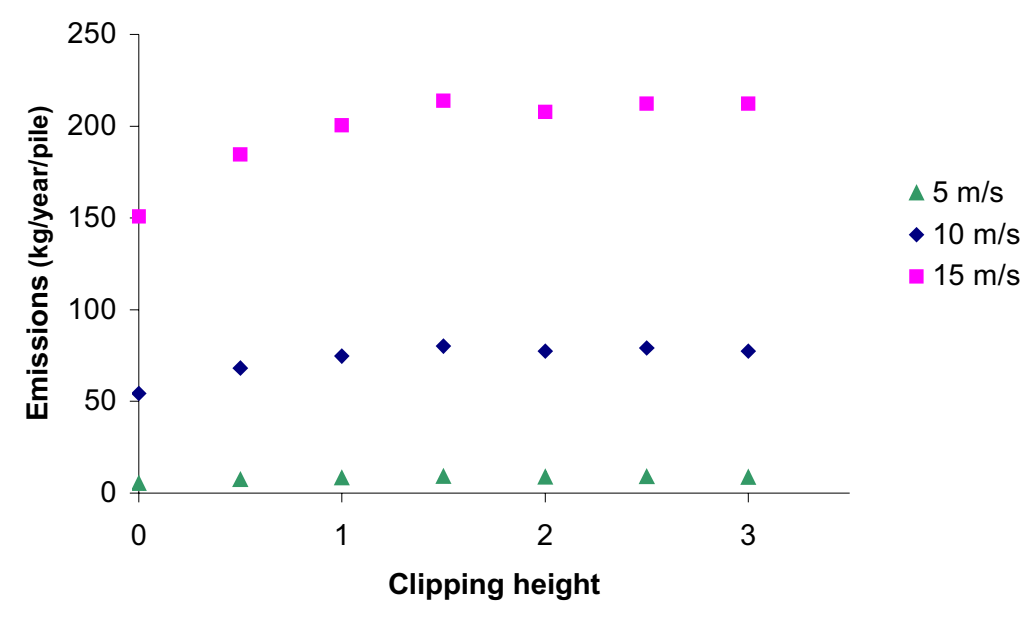

Figure 5: Evolution of the emission factor of a pile as a function of the clipping height for different wind speeds $u_{10}^{+}=5,10$ and $15 \mathrm{~m} / \mathrm{s}$.

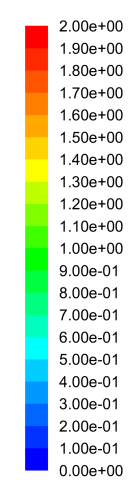

$05235 \mathrm{sil} / 2 \mathrm{~s}$ l'é3mul $2 \mathrm{~m} 2 /$

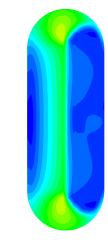

$2 \mathrm{~s} s 1$

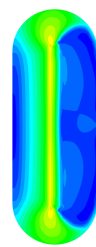

$2 s s 4$

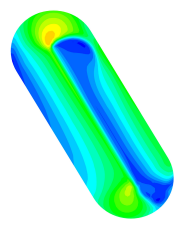

$2 \mathrm{~s} \mathrm{~s}$

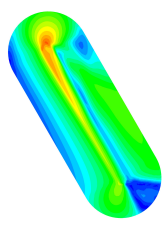

$2 \mathrm{~s} \mathrm{~s}$

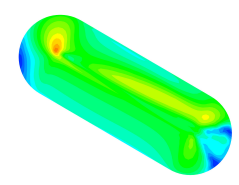

$2 \mathrm{~s} 10$

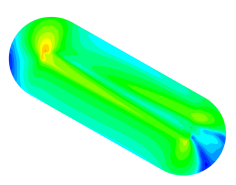

2s s11

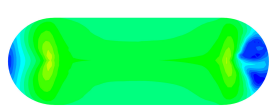

$2 \mathrm{~s} s 12$

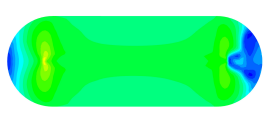

$2 \mathrm{~s} s 13$

Figure 6: Top view of the contours of normalized velocities $u_{s} / u_{r}$ at $25 \mathrm{~cm}$ from the pile surface for the tests 1,4 and 8 to 13 .

have been employed to simulate the wind flow structure over a wide variety of pile geometries and dimensions that can be found on industrial sites [5-7]. In these studies, the wind flow structure was analyzed only for isolated piles configurations without considering their environment on the industrial site. However, the wind erosion process is considerably affected by flow field changes, which are caused by every modifications of the terrain geometry. Nevertheless, till now, at no time the 

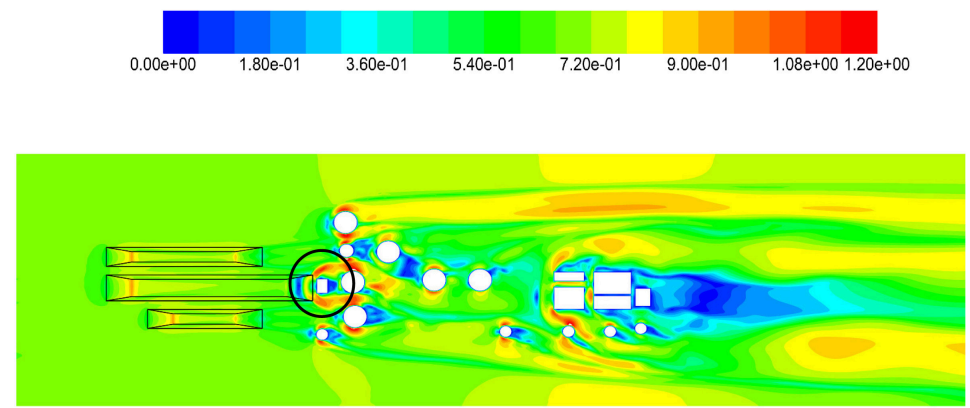

Test $1 \mathrm{~A}$

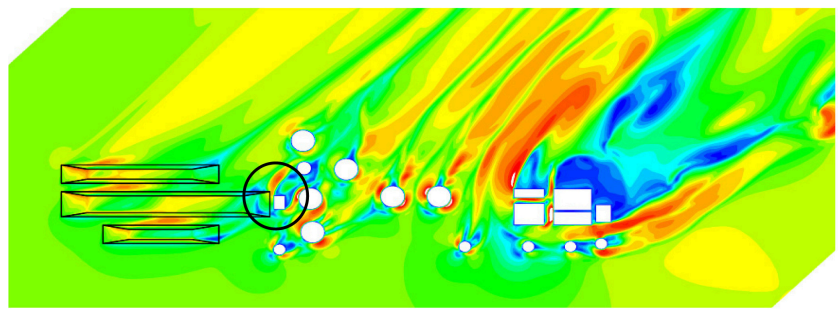

Test 2A

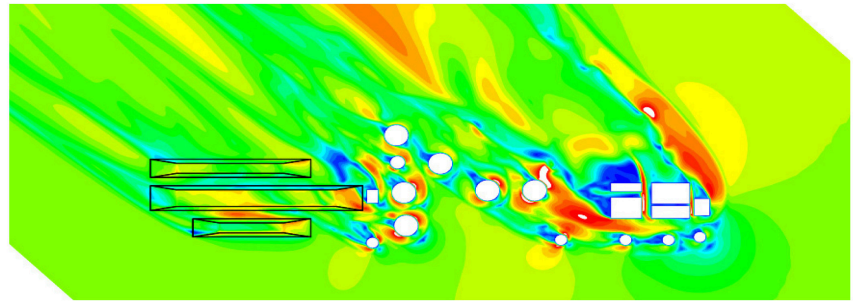

Test 3A

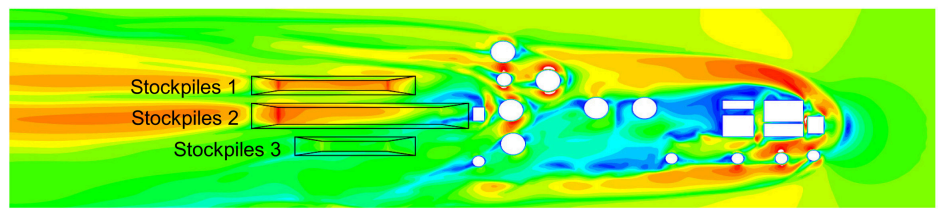

Test 4A

Figure 7: Top view of the normalized velocities contours $u_{s} / u_{r}$ at $25 \mathrm{~cm}$ from the surface for the tests $1 \mathrm{~A}, 2 \mathrm{~A}, 3 \mathrm{~A}$ and $4 \mathrm{~B}$.

effect of the buildings surrounding the stockpiles has been taken into account. In this study, two configurations were tested for various wind flow directions: the first one without representing the buildings and the second one with the surrounding buildings in the calculation domain. 
In a general manner, buildings behave as huge obstacles for the wind flow and perturb largely the dynamic flow structures over stockpiles. The turbulent flow around isolated obstacles has been extensively studied notably by [13]. From their experimental investigations, they have described in detail the flow past a cubical obstacle placed in developed channel flow. When approaching the cube, the flow is decelerates and separates on the upstream corners of the obstacle creating separation vortexes. The main vortex wraps as a horseshoe vortex around the cube. A broad recirculation zone develops downstream of the obstacle. In presence of several obstacles, wakes of the upstream obstacles interferes with the downstream ones. When the obstacles are very close, the wakes interfere and tend to become similar to the wake of a single body.

The effect of the surroundings buildings on the wind speed around the stockpiles is shown in Figure 7 through the contours of the normalized velocity $u_{s} / u_{r}$. In the test $1 \mathrm{~A}$ and $2 \mathrm{~A}$, where the wind first come across the stockpiles, the buildings do not significantly disturb the flow structure over the stockpiles. The low velocity region generated by the flow deceleration upstream the buildings (for the closest to the stockpiles surrounded on Figure 7) interacts with the stockpiles and protects the stockpiles from the wind erosion. The comparison of the results obtained by the numerical simulation for the tests $1 \mathrm{~A}-1 \mathrm{~B}$ and $2 \mathrm{~A}-2 \mathrm{~B}$ show that the presence of the buildings downstream the stockpiles lead to a decrease of the erosion potential of the stockpiles. For the wind directions $\theta=270^{\circ}$ and $315^{\circ}$, the estimation of the dust emissions, calculated by the EPA method, for the tests $1 \mathrm{~A}$ and $2 \mathrm{~A}$ are respectively reduced of $14 \%$ and $2.5 \%$ in comparison with the tests $1 \mathrm{~B}$ and $2 \mathrm{~B}$.

When the flow circulates first of all around the buildings before reaches the stockpiles, the flow structure over the stockpiles is strongly modified. Figure 7 shows, for the test $3 \mathrm{~A}$, that the vortexes generate downstream the closest buildings to the stockpiles interfere with the stockpiles. Near the surface, these vortexes tend to accelerate the wind speed over the stockpiles. In this configuration, the erosion potential estimation increases of $21 \%$ in comparison with the configuration where the buildings are not simulating (test 3B). In the test 4A, the wind flow first comes across the higher buildings which are set very closely. Figure 7 shows that the flow behave like if this group of buildings formed only one big obstacle (right side of the picture), in fact the flow accelerates toward the sides of this large obstacle and a large wake zone is created downstream. Over the stockpiles 1 and 2 the wind speed is very high because these stockpiles are exactly located downstream the vortexes generated by the buildings. The stockpile 3 is, as for it, located in the wake of the buildings, so it is protected from the wind erosion. On average for the test 4A, the erosion potential of the three stockpiles, calculated by the EPA method, has dramatically (90\%) increased by taking into account the presence of the buildings.

For this real configuration of a power plant, the comparison of the results from the two tested configurations (with and without buildings) shows by evidence that the topography of the site exerts large perturbations on the flow structure over the stockpiles. This study shows the necessity to take into account the topography of the industrial site, at least the main buildings and all the significant stockpiles, to enhance the accuracy of the dust emission estimation. 


\section{Conclusion}

In this paper are presented the numerical modeling results of flow structures over various flat-topped stockpiles and over a real configuration of a power plant. The first part of the study consisted in analyzing the flattening effect of the stockpiles crest on dust emissions. This work have revealed that the flattening of the stockpiles crest not allowed to reduce the wind erosion of the stockpiles. The optimal geometrical characteristics of a stockpile, among those tested, minimizing dust emission is a sharped crest stockpile with the main direction perpendicular to the wind flow. In the second part of the study, the presence of the surrounding buildings around the stockpiles was considered for the calculation of the dust emissions rate from the stockpiles. The results of these simulations emphasize the necessity to take into account the topography of the site, at least the main buildings and all the significant stockpiles. In fact, previous studies carried out without regarding the topography of the site led to an inaccurate evaluation of the fugitive dust emissions.

The study reported in this paper improves the understanding of fugitive dust emissions on industrial sites and in mining zones. These findings will allow a more accurate and relevant evaluation of fugitive dust emissions from open storage systems on industrial sites and a better evaluation of its environmental impacts.

\section{Acknowledgements}

This work was carried out with the financial support of ArcelorMittal Dunkerque and Fos-sur-Mer (steelworks in France), EDF Research \& Development and ADEME (the French Agency for Environment and Energy Management).

\section{References}

[1] Neuman, C.M., Lancaster, N. \& Nickling, W., Relations between dune morphology, air flow, and sediment flux over reversing dunes. Sedimentology, $\mathbf{4 4}$, pp. 1103-1113, 1997.

[2] Lancaster, N., Nickling, W., Neuman, C.M. \& Wyatt, V., Sediment flux and airflow on the stoss slope of a barchan dune. Geomorphology, 17, pp. 55-62, 1996.

[3] Parson, D., Wiggs, G., Walker, I., Ferguson, R. \& Garvey, B., Numerical modelling of airflow over an idealised transverse dune. Environmental Modelling and Software, 19, pp. 153-162, 2004.

[4] EPA, Update of fugitive dust emissions factors in ap-42. Midwest Research Institute, Kansas City, MRI No. 8985-K, pp. AP-42 section 11.2, 1988.

[5] Badr, T. \& Harion, J., Numerical modelling of flow over stockpiles: implications on dust emissions. Atmospheric Environment, 39, pp. 5579-5584, 2005.

[6] Badr, T. \& Harion, J., Effect of aggregate storage piles configuration on dust emissions. Atmospheric Environment, 41, pp. 360-368, 2007. 
[7] Torano, J., Rodriguez, R., Diego, I. \& Pelegry, J.R.A., Influence of pile shape on wind erosion cfd emission simulation. Applied mathematical modelling, 31, pp. 2487-2502, 2006.

[8] Stunder, B. \& Arya, S., Windbreak effectiveness for storage pile fugitive dust control: a wind tunnel study. Journal of the air pollution control association, 38, pp. 135-143, 1988.

[9] Menter, F., Two-equation eddy-viscosity turbulence models for engineering applications. AIAA Journal, 32, pp. 1598-1605, 1994.

[10] Neuman, C.M., Lancaster, N. \& Nickling, W., The effect of unsteady winds on sediment transport on the stoss slope of a transverse dune. Sedimentology, 47, pp. 211-226, 2000.

[11] Walker, I. \& Nickling, W., Secondary airflow and sediment transport in the lee of a reversing dune. Earth Surface Processes and Landforms, 24, pp. 438448, 1999.

[12] Walker, I. \& Nickling, W., Dynamics of secondary airflow and sediment transport over and in the lee of transverse dunes. Progress in Physical Geography, 26, pp. 47-75, 2002.

[13] Martinuzzi, R. \& Tropea, C., The flow around surface mounted prismatic obstacle placed in a fully developed channel flow. Journal of Fluid Engineering, 115, pp. 85-92, 1993. 